\title{
Controlo e infinitivos flexionados em português $\mathrm{L2}^{1}$
}

\author{
Alexandra Fiéis \& Ana Madeira \\ CLUNL/NOVA FCSH
}

\begin{abstract}
:
It is known that knowledge of the interpretative properties of the standard inflected infinitive develops late both in L1 and L2 acquisition, and that subject control with most verbs is acquired early in L1. In this study we focus on a context which has not been addressed so far, and investigate how the interpretation of the null subjects of inflected infinitival complements of subject control verbs develops in the interlanguage of Englishand Spanish-speaking learners of L2 European Portuguese. We applied a selection task to intermediate and advanced learners in order to understand whether they differentiate between inflected and uninflected infinitives in these contexts and whether they assign control properties to the inflected infinitive. Our findings show that, although learners accept the occurrence of inflected infinitives with subject control verbs and assign a controlled reading to the infinitival null subject, knowledge of some of the properties of these constructions is delayed, namely, which verbs allow inflected infinitives and what are the interpretative properties of inflected infinitival subjects under different control verbs.
\end{abstract}

Keywords: control, inflected infinitive, null subjects, L2 European Portuguese

Palavras-chave: controlo, infinitivo flexionado, sujeitos nulos, português europeu L2

\section{Introdução}

Neste trabalho investigamos a aquisição das propriedades dos sujeitos nulos de complementos infinitivos flexionados de verbos de controlo de sujeito em português europeu (PE) L2. Procuramos perceber como se desenvolvem as propriedades interpretativas dos sujeitos nulos nestes contextos e se haverá um efeito facilitador da L1, contrastando, para isso, aprendentes de línguas com e sem infinitivos pessoais (espanhol e inglês, respetivamente). Por outro lado, sendo a interpretação dos sujeitos em contextos de controlo determinada por propriedades gramaticais (sintáticas e semânticas) e dependendo a distribuição do infinitivo flexionado (InfFlex) das propriedades lexicais dos verbos de controlo, pretende-se investigar se, de acordo com as predições da Hipótese de Interface (Sorace, 2011), os falantes não-nativos no nível mais avançado de proficiência não apresentam dificuldades nestes domínios.

Sabemos que, na aquisição de PE L1, o controlo de sujeito é adquirido cedo, embora as crianças apresentem atrasos com verbos com um argumento interno objeto indireto (OI) como prometer (Agostinho, 2014). No que diz respeito a InfFlex, embora as suas propriedades morfossintáticas não sejam, em geral, problemáticas, alguns aspetos da sua interpretação desenvolvem-se tardiamente quer na aquisição de L1 (Pires, Rothman \& Santos, 2011) quer na de L2 (Madeira, 2018). Contudo, nenhum dos estudos realizados até ao presente se debruçou sobre a aquisição do InfFlex em contextos de controlo de sujeito.

Com o objetivo de perceber como se desenvolve o conhecimento das propriedades interpretativas dos sujeitos nulos dos complementos infinitivos flexionados nestes contextos na interlíngua de aprendentes de PE

\footnotetext{
${ }^{1}$ Este trabalho foi financiado pela FCT, através do Projeto Estratégico do Centro de Linguística da Universidade Nova de Lisboa UID/LIN/03213/2013. Agradecemos a todos os informantes que participaram no estudo, à Bruna Bragança pela recolha e tratamento dos dados, e a todos os que, de alguma forma, contribuíram para a realização deste estudo.
} 
L2, será utilizada uma tarefa de seleção através da qual se avalia, por um lado, a aceitação de infinitivo flexionado e não flexionado com diferentes verbos de controlo - lamentar, preferir, querer e prometer, isto é, verbos que apresentam comportamentos distintos no que toca à aceitação de InfFlex - e, por outro lado, as interpretações atribuídas aos sujeitos nulos do infinitivo com cada um destes verbos.

O artigo está organizado da seguinte forma: na secção 2, descrevemos as propriedades e a distribuição de InfFlex em PE, considerando em particular os contextos de controlo, e apresentamos alguns dados sobre a aquisição destas propriedades em L1 e L2; na secção 3, apresentamos as questões de investigação; na secção 4, descrevemos a metodologia - a tarefa experimental, os procedimentos e os participantes; na secção 5, descrevemos os resultados; e, finalmente, na secção 6, discutimos os resultados, apresentamos as conclusões, e identificamos algumas questões para trabalho futuro.

\section{O infinitivo flexionado em PE}

Nesta secção, começamos por caracterizar brevemente as propriedades e a distribuição do InfFlex em PE em contextos não controlados (2.1) e em complementos de verbos de controlo (2.2.). Consideramos depois, em 2.3, alguns estudos que se debruçaram sobre o desenvolvimento destas propriedades quer na aquisição de L1 quer na de L2.

\subsection{Propriedades e distribuição}

O InfFlex corresponde tipicamente a uma forma verbal infinitiva que exibe morfologia de pessoa/número e legitima sujeitos referenciais nominativos, realizados ou nulos. Raposo (1987) propõe que, para que a categoria Agr (pessoa/número) possa legitimar sujeitos nominativos nestes contextos, é necessário que ela própria seja marcada casualmente, o que acontece nos casos em que o verbo matriz seleciona um complemento nominal ou o $\mathrm{C}$ infinitivo exibe um operador temporal abstrato (neste caso, é necessário o movimento do verbo de I para $\mathrm{C}$, resultando em inversão do sujeito). Esta condição explica por que, em $\mathrm{PE}, \mathrm{o}$ InfFlex está restrito a alguns dos contextos em que o infinitivo não flexionado ocorre, sendo permitido, por exemplo, em orações adverbiais (1) e em complementos de verbos proposicionais como afirmar e pensar e de verbos factivos como lamentar (2), mas não em complementos de verbos de controlo de sujeito como querer (3):

(1) Os teus pais chegaram cedo para (tu) poderes sair.

(2) O professor afirmou/pensou/lamentou terem (os alunos) saído.

(3) *O professor quer (os alunos) saírem.

O InfFlex distingue-se do infinitivo pessoal que se encontra em línguas como o espanhol, o qual legitima sujeitos nominativos, mas não está associado a morfologia de pessoa e número (e.g., Mensching, 2000; Torrego, 1998). O infinitivo pessoal em espanhol apresenta uma distribuição mais restrita do que o InfFlex em PE, uma vez que está limitado a contextos não selecionados - por exemplo, orações de sujeito e adverbiais -, e o sujeito infinitivo ocorre geralmente em posição pós-verbal (4).

(4) Irse Maribel a Alemania supone que su marido se quede al cuidado de los niños.

(Hernanz Carbó, 1982: 337, apud Mensching, 2000:24)

Pires (2001) mostra que, ao contrário do sujeito PRO de complementos infinitivos não flexionados, o sujeito nulo de orações infinitivas flexionadas (que, de acordo com Raposo, 1987, corresponde à categoria vazia pro) não 
apresenta propriedades de controlo obrigatório (Hornstein, 1999): por exemplo, não requer um antecedente em posição de c-comando na oração matriz (5) e recebe uma leitura estrita em contextos de elipse (6).

(5) a. Os professores $\mathrm{i}_{\mathrm{i}}$ lamentam proj $\mathrm{j}$ termos saído.

b. Os professores $\mathrm{i}_{\mathrm{i}}$ lamentam $\mathrm{PRO}_{\mathrm{i} / *_{\mathrm{j}}}$ ter saído.

(6) a. O António lamenta termos saído e o Rui também (= o Rui lamenta nós termos saído).

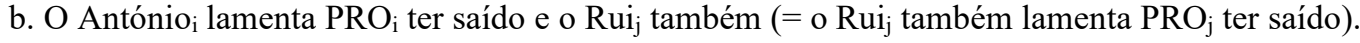

Estas diferenças são atribuídas às diferentes propriedades do núcleo $\mathrm{T}$ em cada uma das construções: assumindo uma análise que deriva os efeitos de controlo do movimento do sujeito infinitivo para uma posição argumental na oração matriz (Hornstein, 1999), Pires (2001) propõe que, enquanto o traço de Caso do sujeito do InfFlex é legitimado in situ pelos traços- $\varphi$ do núcleo $T$ infinitivo, o sujeito do infinitivo não flexionado (que está associado a um núcleo $\mathrm{T}$ defetivo) é obrigado a mover-se para uma posição na matriz para verificação de Caso, apresentando, assim, as propriedades que estão tipicamente associadas ao controlo obrigatório.

\subsection{Infinitivo flexionado e controlo}

O InfFlex pode ocorrer opcionalmente em complementos de verbos de controlo obrigatório, como é o caso de verbos de controlo de objeto como convencer (Raposo, 1989). Nestes complementos, InfFlex apresenta propriedades de controlo obrigatório, como se pode observar na frase em (7), em que o sujeito infinitivo é controlado (exaustiva ou parcialmente) pelo objeto direto matriz os meninos e não pode ser realizado foneticamente.

(7) Ele en $_{\mathrm{i}}$ convenceu os meninos $\mathrm{j}$ a (*eles $)_{\mathrm{j} / \mathrm{i}+\mathrm{j}} \mathbf{i r}(\mathbf{e m})$ ao cinema.

Como Sheehan $(2014,2018)$ mostra, e ao contrário do que é assumido em Raposo (1987), o InfFlex pode também ocorrer nos complementos de alguns verbos de controlo de sujeito com um sujeito nulo que é controlado pelo sujeito matriz. Contudo, existe muita variabilidade nos juízos dos falantes nativos de PE no que diz respeito à aceitação do InfFlex nestes contextos. Três fatores, em particular, condicionam a aceitabilidade do InfFlex: o tipo de verbo, o tipo de leitura de controlo e a localidade da relação de controlo.

Relativamente ao tipo de verbo, Sheehan (2018) sugere uma possível correlação entre o fenómeno de reestruturação e a possibilidade de InfFlex, na medida em que a maioria dos falantes nativos de PE rejeita o InfFlex com verbos de reestruturação como querer - embora a sua ocorrência com estes verbos esteja atestada em dados de produção (e.g., Fiéis \& Madeira, 2017; Madeira \& Fiéis, 2019) e de juízos de aceitabilidade (Pires, Rothman \& Santos, 2011) -, mas aceita-o com verbos que não permitem (ou desfavorecem) reestruturação, como é o caso de prometer e preferir. Também Gonçalves et al (2014) correlacionam a possibilidade de InfFlex com tempo independente na encaixada, um contexto em que a reestruturação não é esperada.

Com estes verbos, o grau de aceitabilidade depende do tipo de leitura de controlo. Por exemplo, com verbos transitivos como preferir, Sheehan (2014, 2018) mostra, com base em dados de juízos de aceitabilidade, que os falantes nativos de PE tendem a rejeitar a flexão quando a leitura é de controlo exaustivo (ou seja, quando há correferência entre o sujeito da oração matriz e o da oração infinitiva), aumentando a aceitabilidade do InfFlex nos casos em que o sujeito da oração infinitiva recebe uma leitura de controlo parcial (isto é, quando o sujeito da matriz está incluído na referência do sujeito da oração infinitiva) (veja-se também Gonçalves, Santos \& Duarte, que, embora com base num menor número de ocorrências e 
num teste de juízos de aceitabilidade menos formal, chegam a uma conclusão idêntica). De igual modo, com um verbo ditransitivo como prometer, a leitura de controlo parcial recebe uma aceitação mais elevada do que a leitura de controlo exaustivo.

De modo geral, a presença de um argumento entre o sujeito da matriz e o sujeito da infinitiva leva a uma maior aceitabilidade do InfFlex, o que significa que a ocorrência de InfFlex é sempre considerada mais aceitável com prometer, que permite o estabelecimento de uma relação de controlo não local, do que com preferir, com o qual a relação de controlo é necessariamente local. Os exemplos abaixo são retirados de Sheehan (2018:32-34); as percentagens que se apresentam referem-se às taxas de aceitação de infinitivo não flexionado e flexionado obtidas numa tarefa de juízos de aceitabilidade aplicada a falantes nativos de PE.

(8) Controlo de sujeito exaustivo local (não flexionado 100\%; flexionado 4\%): Preferíamos receber(*mos) um salário maior.

(9) Controlo de sujeito parcial local com preferir (não flexionado $83 \%$; flexionado $42 \%$ ): O João $\mathrm{O}_{i}$ preferia $\mathrm{PRO}_{\mathrm{i}+}$ reunir(\%em)-se mais tarde.

(10) Controlo de sujeito parcial local com querer (flexionado 9\%): *O João queria reunirem-se mais cedo.

(11) Controlo de sujeito exaustivo não local (não flexionado $100 \%$; flexionado $47 \%$ ): Prometemos à professora chegar(\%mos) a tempo.

(12) Controlo de sujeito parcial local com preferir (não flexionado $70 \%$; flexionado $95 \%$ ): $\mathrm{O}$ Pedro prometeu à Ana reunir(em)-se em Braga.

Nestes contextos, o sujeito da oração infinitiva flexionada exibe propriedades típicas de controlo obrigatório (Modesto, 2010; Sheehan, 2014, 2018): por exemplo, não pode ser realizado lexicalmente (13), requer um antecedente local que o c-comande (14) e recebe uma leitura não-estrita (sloppy) com elipse de VP (15):

(13) *O Pedro prefere os meninos reunirem-se mais cedo.

(14) a. *O Pedro acha que eu preferia reunirem-se mais cedo. b. \%[A chefe do João $\left.{ }_{i}\right]_{j}$ preferia reunirem-se sem ele $e_{i}$. (Sheehan, 2018:40)

c. *[A chefe do João $]_{j}$ j preferia reunirem-se sem elaj. (idem:41)

(15) a. \%O João preferia reunirem-se de manhã e a Maria também preferia (*mas sem ela). (idem:40)

Verbos como lamentar (2.1), que selecionam complementos de InfFlex, selecionam igualmente infinitivo não flexionado, funcionando, neste caso, como verbos de controlo de sujeito. Note-se que os sujeitos dos complementos de InfFlex destes verbos, aos quais se atribui, tipicamente, uma leitura de referência livre, podem também, opcionalmente, receber uma leitura de controlo exaustivo ou parcial (17), idêntica à que recebem com o infinitivo não flexionado (16).

(16) Os meninos $\mathrm{i}_{\mathrm{i}}$ lamentam $\mathrm{PRO}_{\mathrm{i} / \mathrm{i}+\mathrm{j}}$ reunir-se tão cedo.

(17) a. Os meninos ${ }_{i}$ lamentam $[-]_{i / i+j / j}$ reunirem-se tão cedo.

b. O menino ${ }_{i}$ lamenta $[-]_{i+j / j}$ reunirem-se tão cedo.

Em suma, InfFlex pode ocorrer em contextos de controlo obrigatório, tal como o infinitivo não flexionado. Nestes contextos, e considerando em particular os verbos de controlo de sujeito, o InfFlex não apresenta exatamente a mesma distribuição que o infinitivo não flexionado. $\mathrm{Na}$ tabela 1 , apresenta-se uma 
síntese da distribuição de InfFlex com os verbos lamentar, preferir, querer e prometer, os verbos que são investigados no presente estudo.

\begin{tabular}{|l|c|c|c|}
\hline \multicolumn{1}{|c|}{ Verbo } & Controlo exaustivo & Controlo parcial & Referência livre \\
\hline lamentar & $\checkmark$ & $\checkmark$ & $\checkmark$ \\
\hline preferir & $*$ & $\%$ & $*$ \\
\hline querer & $*$ & $*$ & $*$ \\
\hline prometer & $\%$ & $\checkmark$ & $*$ \\
\hline
\end{tabular}

Tabela 1: Distribuição de InfFlex com diferentes verbos de controlo de sujeito

Uma vez que, à semelhança do infinitivo não flexionado, o InfFlex apresenta, nestes contextos de controlo, propriedades de controlo obrigatório, Gonçalves, Santos \& Duarte (2014) propõem que estes infinitivos flexionados são infinitivos de controlo, ou seja, infinitivos cujo sujeito é a categoria vazia PRO. A existência destas formas, que Gonçalves, Santos \& Duarte (2014) designam de "pseudo-infinitivos flexionados", comprova que a presença de flexão de pessoa/número no infinitivo não está necessariamente associada à capacidade de legitimar sujeitos com caso nominativo. De acordo com a análise proposta por estas autoras (seguindo Landau, 2000), assume-se que, nestes infinitivos, a flexão de pessoa/número corresponde ao spell-out de uma operação de AGREE entre a categoria funcional $\mathrm{T}$ da oração infinitiva e um núcleo funcional mais alto.

\subsection{Dados de aquisição}

A investigação que se tem debruçado sobre a aquisição do InfFlex (não controlado) em PE L1 tem demonstrado que este é um fenómeno gramatical que emerge cedo, mas está sujeito a desenvolvimento tardio. Assim, em produção espontânea, começa por aparecer em adverbiais finais, cerca dos 2 anos de idade, e em completivas selecionadas por verbos percetivos e causativos, por volta dos 3 anos (Santos et al., 2013). De acordo com Pires, Rothman \& Santos (2011), as crianças só atingem conhecimento pleno das propriedades morfossintáticas do InfFlex por volta dos 6-7 anos de idade e das suas propriedades interpretativas por volta dos 9 anos. Em idades mais precoces, as crianças apresentam uma preferência por leituras de controlo para o sujeito nulo das orações de InfFlex.

No que diz respeito a PE L2, Madeira (2018) investigou a aquisição de propriedades morfossintáticas e interpretativas do InfFlex por falantes nativos de espanhol e de chinês, com base em dados de produção e de juízos de aceitabilidade. Confirmando as predições da Hipótese de Interface (e.g., Sorace, 2011), segundo a qual as propriedades interpretativas do sujeito (dependentes de conhecimento que está na interface sintaxe/discurso) deverão ser mais problemáticas do que as propriedades estritamente gramaticais, verificouse que aprendentes num nível avançado de proficiência não exibiam conhecimento pleno das propriedades interpretativas dos sujeitos infinitivos (em particular, dos sujeitos realizados), mas distinguiam claramente o infinitivo não flexionado do flexionado e demonstravam conhecimento das propriedades e da distribuição específicas deste (por exemplo, o conhecimento de que existe uma associação entre a presença de marcas morfológicas de pessoa/número e a realização de sujeitos nominativos), embora este conhecimento se desenvolvesse tardiamente. Os resultados revelaram também a existência de diferenças entre os dois grupos de falantes não-nativos, indicando um efeito de influência da L1 que se manifestava de forma diferente para os dois tipos de propriedades. Assim, o atraso no desenvolvimento das propriedades morfossintáticas do InfFlex observou-se, em particular, no grupo de falantes de espanhol, que, até tarde, apresentava dificuldades na distinção entre o infinitivo flexionado e o não flexionado: estas dificuldades manifestavam-se, por exemplo, pela aceitação de InfFlex em contextos em que apenas infinitivos não flexionados podem ocorrer. 
Tal sugere que a existência, na L1 destes aprendentes, de um infinitivo pessoal - uma construção que é superficialmente semelhante ao InfFlex, mas que se caracteriza por propriedades gramaticais distintas poderá dificultar a aquisição das propriedades gramaticais do InfFlex em PE. Por outro lado, o atraso no desenvolvimento das propriedades interpretativas dos sujeitos do InfFlex ocorreu sobretudo no grupo de falantes de chinês; neste caso, a existência, em espanhol, de sujeitos pronominais (nulos e realizados) com propriedades idênticas às dos sujeitos do PE parece facilitar a aquisição destas propriedades para o grupo de falantes de espanhol. Neste estudo, não se observaram quaisquer dificuldades na atribuição de uma interpretação de controlo obrigatório aos sujeitos de infinitivos não flexionados em nenhum dos dois grupos de aprendentes.

Não existem, até ao presente, estudos sobre a aquisição do infinitivo flexionado controlado em PE L2. Relativamente a L1, sabemos que, de modo geral, as construções de controlo (de sujeito e de objeto) são adquiridas cedo, embora as crianças apresentem atrasos na interpretação de verbos de controlo de sujeito com um argumento interno OI como prometer (Agostinho, 2014). Dados de produção induzida (Santos, Gonçalves \& Hyams, 2016) mostram que, por volta dos 5 anos, as crianças produzem InfFlex com verbos de controlo de objeto como ensinar $a$; nesta idade, as crianças também produzem InfFlex com verbos de controlo de sujeito como querer e conseguir, com sujeito realizado não-controlado. De maneira geral, o conhecimento das propriedades e da distribuição do InfFlex em contextos de controlo parece desenvolver-se tarde.

\section{Questões de investigação}

Como foi referido na secção 2, no PE, o InfFlex pode ocorrer em alguns contextos de controlo obrigatório, como é o caso dos complementos de verbos como preferir e prometer, com um sujeito não realizado lexicalmente que é controlado pelo sujeito matriz (Sheehan, 2014, 2018). Porém, os juízos dos falantes nativos caracterizam-se por uma grande variabilidade. No que toca a aquisição, vimos que, embora as propriedades morfossintáticas do InfFlex não sejam, em geral, problemáticas, alguns aspetos da sua interpretação desenvolvem-se tardiamente quer em L1 (Pires, Rothman \& Santos, 2011) quer em L2 (Madeira, 2018). Da escassa investigação realizada até ao presente sobre a aquisição de construções de controlo e, em particular, do InfFlex em contextos de controlo de sujeito, sabemos que as crianças adquirem cedo as propriedades dos verbos de controlo de sujeito em PE L1 (com exceção de prometer; Agostinho, 2014); contudo, tanto a distribuição do InfFlex com estes verbos como as restrições à sua ocorrência são adquiridas mais tarde. Este é um fenómeno que não foi ainda estudado no PE L2.

Neste trabalho, investigamos as propriedades dos sujeitos nulos de complementos infinitivos flexionados e não flexionados de verbos de controlo de sujeito na interlíngua de falantes nativos de espanhol, uma língua que, embora não possua InfFlex, permite infinitivos pessoais num conjunto restrito de contextos (como referido em 2.1), e de falantes nativos de inglês, que não possui InfFlex nem infinitivos pessoais. Nestes contextos, a interpretação dos sujeitos infinitivos é determinada por propriedades gramaticais (sintáticas e semânticas) e a distribuição de InfFlex depende das propriedades lexicais dos verbos de controlo. Deste modo, a aquisição de InfFlex controlado implica o desenvolvimento do conhecimento, por um lado, de que a presença de morfologia de pessoa/número no infinitivo pode estar dissociada das propriedades sintáticas características de InfFlex e, por outro lado, de quais são os verbos com que este InfFlex é permitido. De acordo com a Hipótese de Interface (Sorace, 2011), prediz-se, pois, que os falantes não-nativos no nível mais avançado de proficiência não apresentem dificuldades relativas às propriedades do InfFlex controlado, uma vez que estas envolvem módulos da gramática (ou seja, são propriedades de interfaces internas, que a Hipótese de Interface prediz serem menos problemáticas do que as propriedades que envolvem a interface com domínios cognitivos externos à gramática, como é o caso de propriedades na interface sintaxe-discurso). Procuramos, então, responder às seguintes questões: 
1. Os aprendentes de PE L2 desenvolvem conhecimento de que o InfFlex pode ocorrer em determinados contextos de controlo (cf. Tabela 1) e de que, nestes contextos, apresenta diferenças em relação ao infinitivo não flexionado?

2. Com verbos de controlo que permitem InfFlex, este apresenta propriedades características dos infinitivos de controlo?

3. Observa-se um efeito de L1 na aquisição destas propriedades?

4. Observa-se um efeito de desenvolvimento na aquisição destas propriedades?

\section{Metodologia}

\subsection{Tarefa e procedimentos}

De modo a responder às questões de investigação elencadas na secção anterior, conduzimos uma tarefa de seleção, com 32 itens de teste (e 32 distratores). Os verbos utilizados nas orações de InfFlex são predicados coletivos (reunir-se e encontrar-se), que exigem um sujeito plural, e o infinitivo apresenta flexão de $3^{\text {a }}$ pessoa do plural. As variáveis testadas foram as seguintes: a) tipo de verbo: lamentar / preferir / querer / prometer; b) tipo de infinitivo: flexionado / não flexionado; c) tipo de sujeito matriz: $3 \mathrm{sg}$ (favorece leitura de controlo parcial) / $3 \mathrm{pl}$ (favorece leitura de controlo exaustivo).

Os participantes realizaram a tarefa online, sem limite de tempo. As frases eram apresentadas uma a uma, estando a ordem de apresentação aleatorizada. Em (18) e (19), mostram-se dois exemplos de itens de teste com infinitivo flexionado e não flexionado, respetivamente. Em cada item, apresentava-se primeiro a frase e pedia-se ao participante que indicasse se considerava a frase aceitável ou não (18a) e (19a). Em caso afirmativo, pedia-se que selecionasse a interpretação ou interpretações mais adequadas para o sujeito do InfFlex: controlo exaustivo, controlo parcial, antecedente split (apenas com prometer) ou referência livre (18b) e (19b).

(18) a. Os alunos querem reunirem-se no pavilhão desportivo.

Esta frase é aceitável em português?

O Sim.

O Não.

b. Na frase "Os alunos querem reunirem-se no pavilhão desportivo", quem é que se vai reunir no pavilhão desportivo?

O Os alunos.

Os alunos e outras pessoas não mencionadas na frase.

Outras pessoas não mencionadas na frase.

(19) a. As amigas preferem encontrar-se no café.

Esta frase é aceitável em português?

O Sim.

O Não.

b. Na frase "As amigas preferem encontrar-se no café", quem é que se encontra no café?

As amigas.

As amigas e outras pessoas não mencionadas na frase.

Outras pessoas não mencionadas na frase. 


\subsection{Participantes}

Como foi referido em 4, os participantes são 35 aprendentes de PE, de nível intermédio e avançado, falantes nativos de espanhol e inglês. Foram escolhidos aprendentes destas duas línguas para investigar as propriedades dos sujeitos nulos de complementos infinitivos (flexionados e não flexionados) de verbos de controlo de sujeito, uma vez que, em espanhol, não há InfFlex (embora haja infinitivos pessoais num conjunto restrito de contextos; cf. Mensching, 2000), e, em inglês, não há InfFlex nem infinitivos pessoais. Saliente-se ainda que, de acordo com a informação fornecida pelos participantes, metade do grupo de inglês L1 tem o espanhol como L2 (avançado/quase nativo). O estudo inclui ainda um grupo de controlo de falantes nativos de PE. As características dos participantes são apresentadas na tabela 2.

\begin{tabular}{|l|c|}
\hline \multicolumn{1}{|c|}{ L1 } & $\begin{array}{c}\text { Idades (média e desvio } \\
\text { padrão) }\end{array}$ \\
\hline Espanhol intermédio $(\mathrm{n}=13)$ & $19-32, \mathrm{M}=22.8, \mathrm{DP}=3.3$ \\
\hline Espanhol avançado $(\mathrm{n}=8)$ & $18-23 \mathrm{M}=19.6 \mathrm{DP}=2.4$ \\
\hline Inglês intermédio $(\mathrm{n}=6)$ & $18-22 \mathrm{M}=19 \mathrm{DP}=1.6$ \\
\hline Inglês avançado $(\mathrm{n}=8)$ & $15-29 \mathrm{M}=20.3 \mathrm{DP}=4.3$ \\
\hline Controlos $(\mathrm{n}=15)$ & $18-44 \mathrm{M}=24.7$ \\
\hline
\end{tabular}

Tabela 2: Participantes no estudo

\section{Resultados}

Nesta secção, descrevemos os resultados da tarefa, considerando as três variáveis linguísticas: o tipo de infinitivo (flexionado e não flexionado); o tipo de verbo (lamentar, preferir, querer e prometer); e o tipo de contexto (sujeito matriz singular e plural).

Como se mostra nos gráficos 1 e 2, observa-se uma aceitação generalizada dos dois infinitivos com todos os verbos. Excetua-se a aceitação de InfFlex com preferir e querer, que se traduz na baixa aceitação por parte dos controlos e na menor aceitação por parte dos não-nativos (sobretudo do grupo de espanhol L1 com querer) comparativamente a lamentar e prometer.

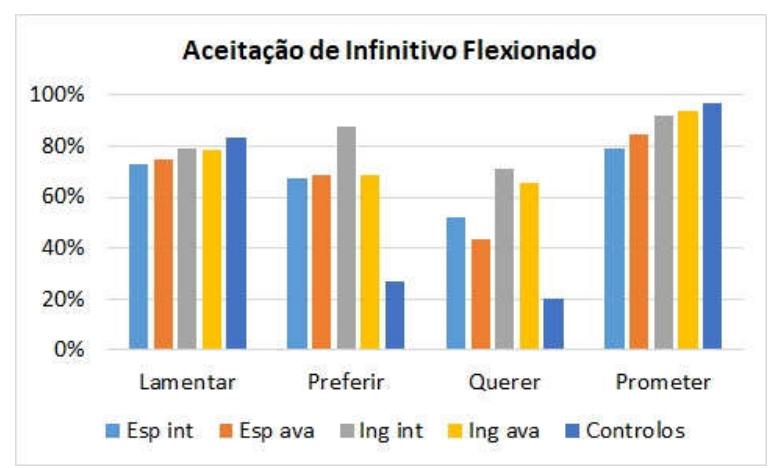

Gráfico 1: Aceitação de infinitivo flexionado

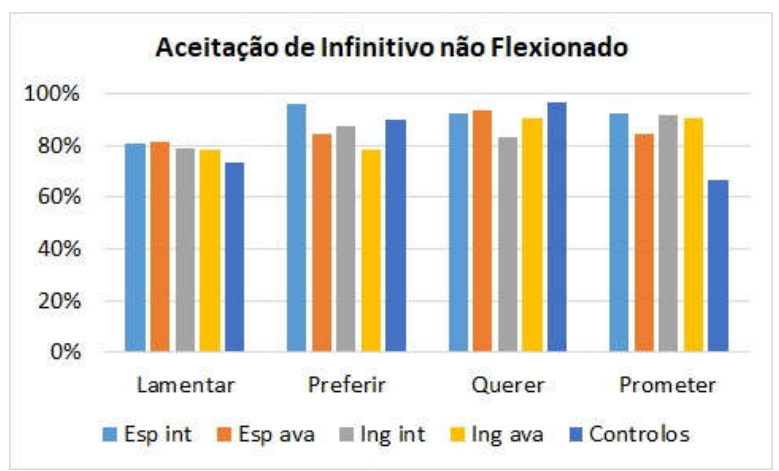

Gráfico 2: Aceitação de infinitivo não flexionado 
Uma vez que se verifica a aceitação generalizada do infinitivo não flexionado com todos os verbos, vamos focar a nossa atenção apenas na aceitação de InfFlex por tipo de sujeito (singular e plural), e por verbo (gráficos de 3 a 6).

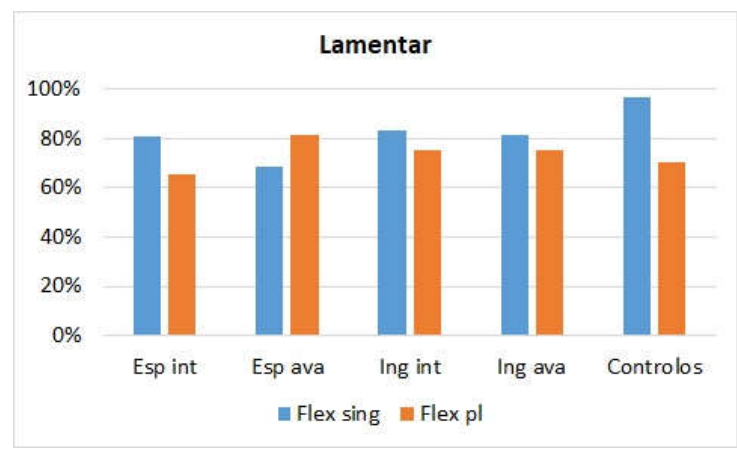

Gráfico 3: Aceitação de infinitivo flexionado com

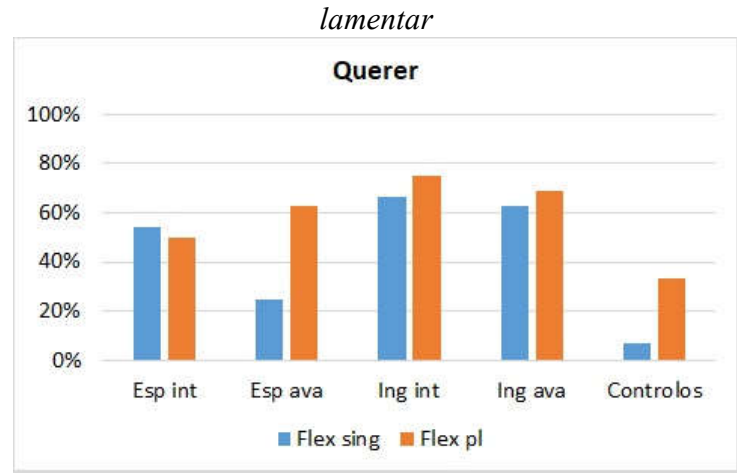

Gráfico 5: Aceitação de infinitivo flexionado com querer

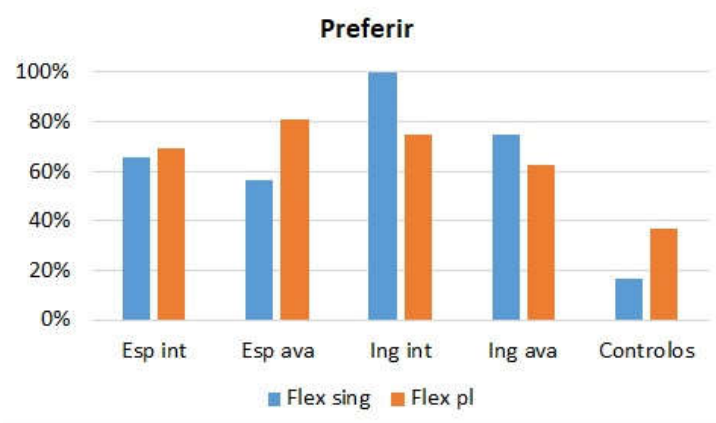

Gráfico 4: Aceitação de infinitivo flexionado com preferir

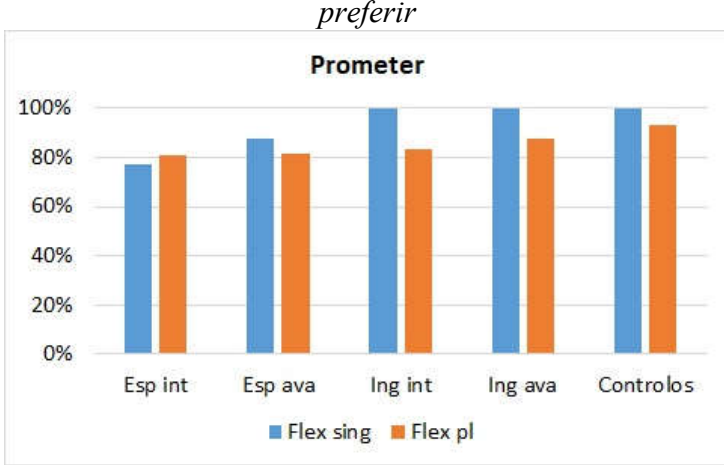

Gráfico 6: Aceitação de infinitivo flexionado com prometer

Confirmam-se as tendências observadas nos resultados globais. Ou seja, há um contraste entre preferir e querer, por um lado, e lamentar e prometer, por outro, na aceitação de InfFlex, sobretudo com sujeito matriz singular; este contraste é particularmente claro no caso dos falantes nativos. Comparando os grupos intermédio e avançado de cada L1, observa-se ainda uma diminuição da aceitação de InfFlex com querer no grupo de espanhol e com prometer no grupo de inglês à medida que a proficiência aumenta.

Analisamos agora as leituras preferenciais, cruzando ainda os tipos de infinitivo (flexionado vs. não flexionado) e o tipo de sujeito matriz (singular ou plural). As taxas de seleção de cada uma das leituras, que se apresentam nos gráficos 7 a 14, foram calculadas com base no número total de itens por condição.

Começamos com os contextos em que o sujeito matriz é singular, nos gráficos 7 a 10 . 


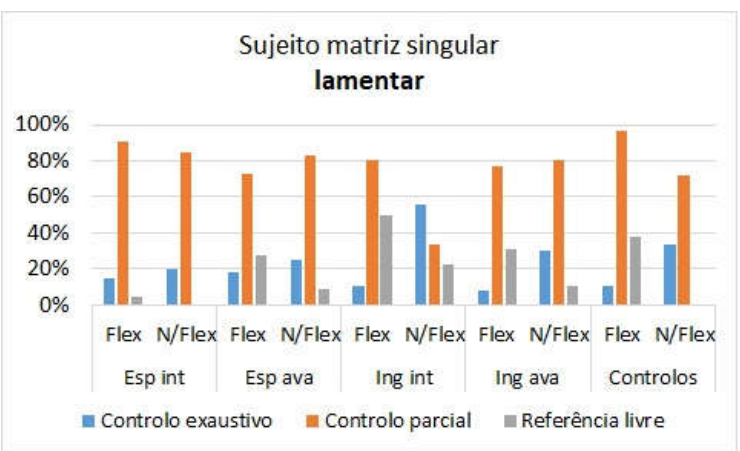

Gráfico 7: Leituras com lamentar - sujeito singular

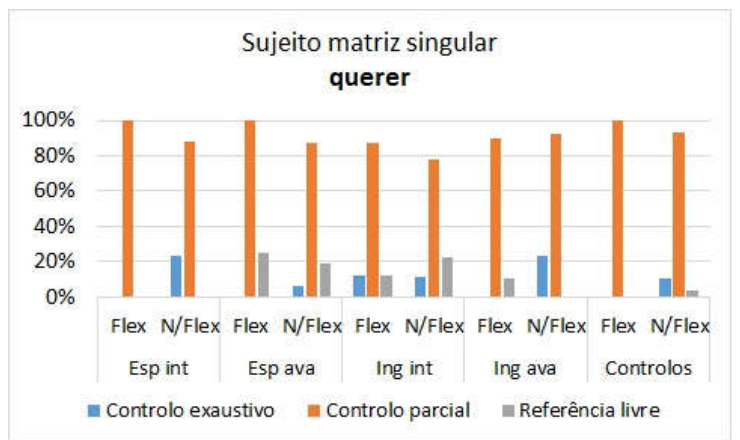

Gráfico 9: Leituras com querer - sujeito singular

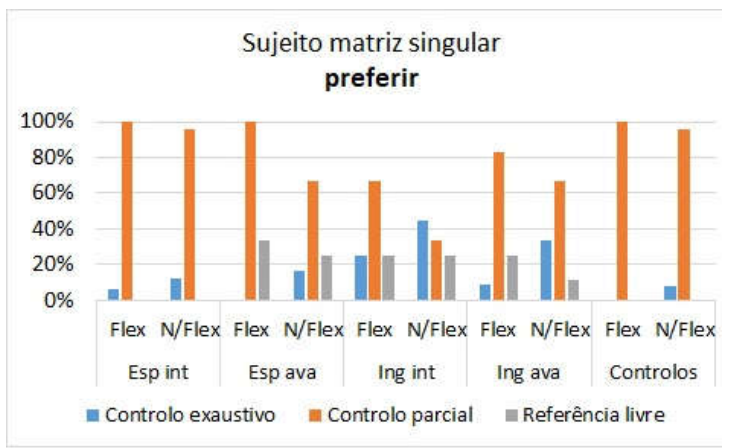

Gráfico 8: Leituras com preferir - sujeito singular

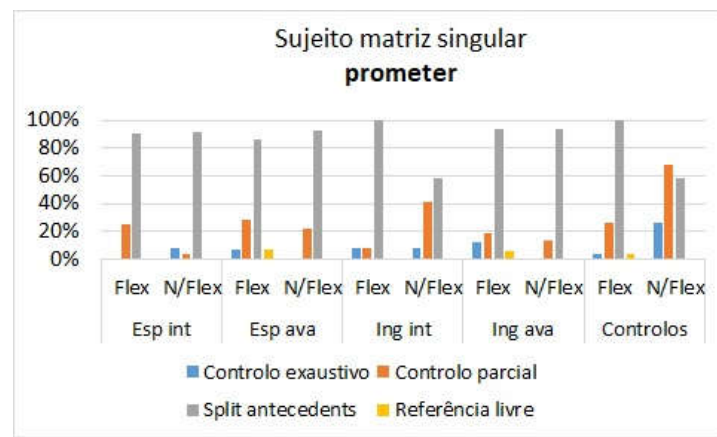

Gráfico 10: Leituras com prometer - sujeito singular

Considerando, em primeiro lugar, o InfFlex, sendo o sujeito singular e o verbo da oração infinitiva um predicado coletivo, esperava-se uma preferência por uma leitura de controlo parcial com todos os verbos. Verifica-se, efetivamente, que esta é a leitura preferida, em todos os grupos, com todos os verbos (com exceção de prometer), observando-se taxas de aceitação de leitura de controlo exaustivo e de referência livre baixas com todos os verbos (neste último caso, sobretudo com querer e prometer).

No caso de prometer, a leitura preferencial é uma leitura de split, isto é, uma leitura na qual a referência do sujeito da oração infinitiva corresponde ao sujeito e ao objeto (e.g., Eu $u_{i}$ prometi ao João ${ }_{j}[-]_{i+j}$ irmos ao $_{\text {a }}$ cinema).

Com os verbos preferir e querer, observam-se diferenças entre os grupos: embora a leitura preferida seja a de controlo parcial para todos os grupos, observam-se baixas taxas de aceitação desta leitura no grupo de falantes nativos (com ambos os verbos) e no grupo de espanhol avançado (apenas com querer), como resultado da rejeição de InfFlex nestes contextos.

Observam-se tendências idênticas com o infinitivo não flexionado, ou seja, a preferência pela leitura de controlo parcial com todos os verbos e as baixas taxas de aceitação de leitura de controlo exaustivo e de referência livre com todos os verbos. No entanto, no caso de prometer, observa-se que os falantes não-nativos preferem uma leitura split, aspeto em que se distinguem dos controlos, que optam de forma igual por controlo parcial. Há a salientar também algumas diferenças entre os grupos com preferir: os controlos e o grupo de espanhol optam por controlo parcial, contrariamente ao grupo de inglês, que não parece ter uma leitura preferida; e o grupo de espanhol avançado diminui a leitura de controlo parcial comparativamente ao grupo intermédio.

vemos agora o que sucede nos contextos com sujeito matriz plural, nos gráficos 11 a 14 . 


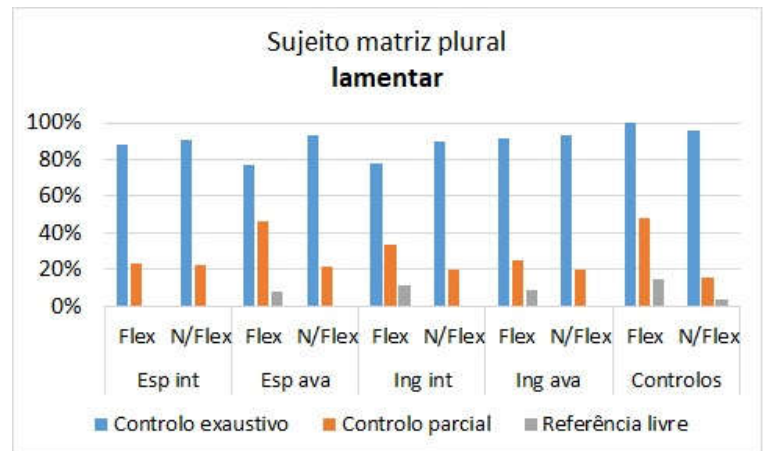

Gráfico 11: Leituras com lamentar - sujeito plural

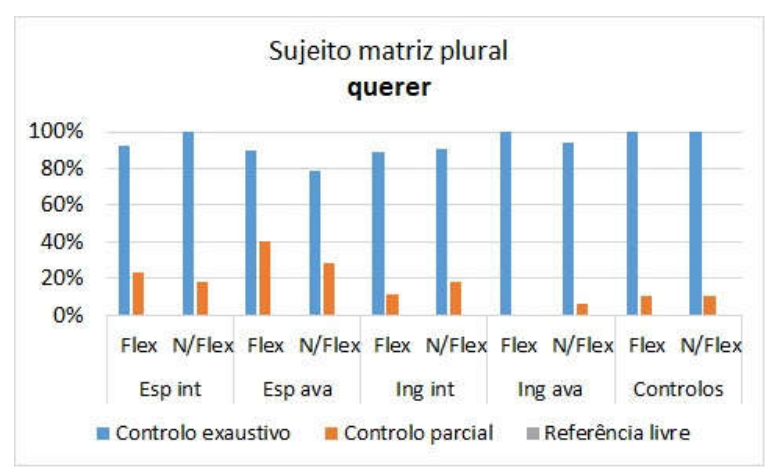

Gráfico 13: Leituras com querer - sujeito plural

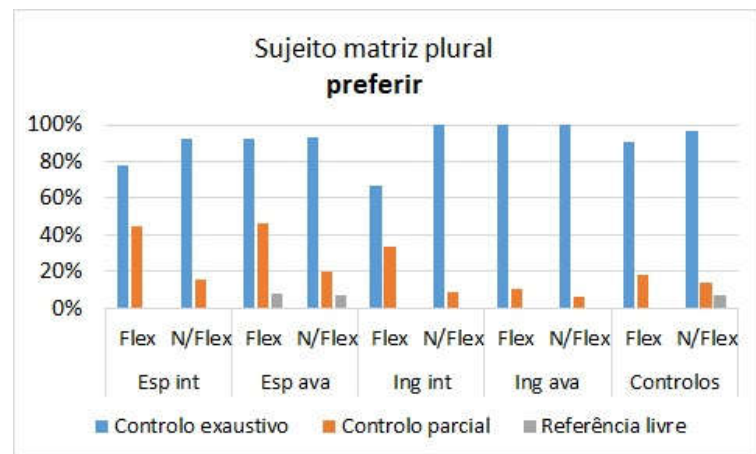

Gráfico 12: Leituras com preferir - sujeito plural

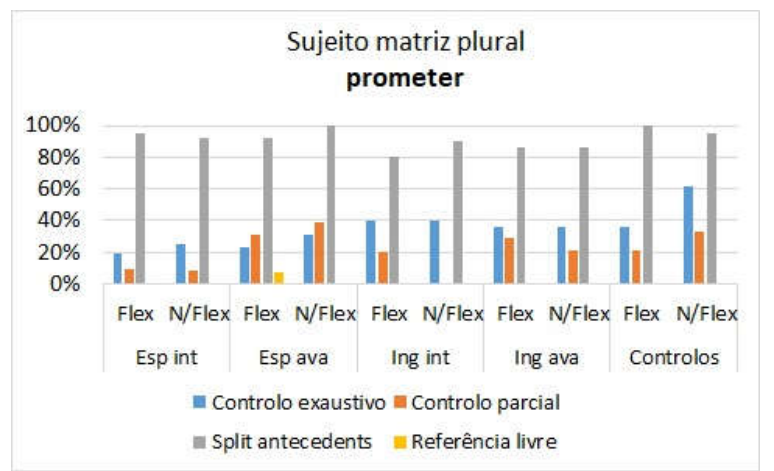

Gráfico 14: Leituras com prometer - sujeito plural

Com sujeito matriz plural, uma vez que o verbo da oração infinitiva, como vimos, é um predicado coletivo e o infinitivo se encontra na $3^{\text {a }}$ pessoa do plural, esperava-se uma preferência por uma leitura de controlo exaustivo com todos os verbos. Esta é, de facto, a tendência que se verifica, não apenas com o infinitivo flexionado, mas também com o não flexionado. A única exceção, novamente, observa-se com o verbo prometer, que favorece uma leitura split com ambos os infinitivos, em todos os grupos. Obtêm-se taxas baixas de leituras de referência livre com todos os verbos.

Em síntese, de maneira geral, as leituras preferidas, para todos os grupos, são as leituras de controlo exaustivo (com sujeito plural) e de controlo parcial (com sujeito singular), quer no infinitivo flexionado quer no não flexionado. O único verbo que apresenta um comportamento distinto é prometer, que favorece a interpretação split, independentemente de número. Em geral, os participantes (tanto os nativos como os nãonativos) não atribuem uma leitura de referência livre ao sujeito do InfFlex, embora se observem taxas de aceitação ligeiramente mais elevadas desta leitura, na condição de sujeito singular, com os verbos lamentar e preferir nos grupos de aprendentes (com exceção do grupo de espanhol intermédio) e com o verbo lamentar no grupo de falantes nativos.

\section{Discussão e conclusões}

Retomamos as questões de investigação, tendo em conta os resultados apresentados na secção anterior. 
1. Os aprendentes de PE L2 desenvolvem conhecimento de que o InfFlex pode ocorrer em determinados contextos de controlo e de que, nestes contextos, apresenta diferenças em relação ao infinitivo não flexionado?

Observa-se aceitação generalizada tanto de infinitivo flexionado como não flexionado com todos os verbos de controlo que permitem InfFlex, apesar de não ser claro se os falantes não-nativos têm conhecimento das restrições à ocorrência de infinitivo flexionado nestes contextos ou se simplesmente não distinguem os dois infinitivos. Isto acontece porque os resultados são ambíguos: os aprendentes apresentam taxas de aceitação de InfFlex elevadas em contextos em que o grupo de controlo apresenta uma fraca aceitação, isto é, com os verbos preferir e querer, apesar de a aceitação de InfFlex com estes dois verbos - e, em particular, com querer - ser, em geral, mais baixa do que com lamentar e prometer.

Os resultados com o verbo lamentar também não permitem retirar conclusões seguras quanto à existência de uma distinção entre infinitivos flexionados e não flexionados nas gramáticas dos aprendentes. Recorde-se que este é um contexto que permite quer a leitura controlada quer a leitura de referência livre para o sujeito da infinitiva. No entanto, os aprendentes manifestam uma preferência clara pela leitura de controlo com este verbo. Contudo, esta é também a leitura preferencial do grupo de falantes nativos, apesar de o verbo lamentar ser o único verbo com que este grupo aceita a leitura de referência livre na condição de sujeito singular. Isto indica que, embora esta não seja a leitura preferida (o que poderá estar relacionado com a presença de um predicado coletivo na oração infinitiva), é claramente uma leitura possível para os falantes nativos. Além disso, com exceção do grupo de espanhol intermédio, que não permite uma leitura de referência livre com lamentar, todos os grupos de aprendentes estabelecem uma distinção clara entre lamentar (com o qual aceitam a leitura referencial) e querer/prometer (com os quais excluem esta leitura), à semelhança do grupo de controlo. Porém, não parece haver diferenças na aceitação de leitura de referência livre entre lamentar e preferir, distinguindo-se, aqui, os grupos de aprendentes do grupo de falantes nativos, que diferencia claramente os dois verbos, rejeitando totalmente a possibilidade de uma leitura referencial com preferir (note-se, todavia, que alguns falantes nativos aceitam esta leitura, de acordo com Sheehan, 2018).

2. Com verbos de controlo que permitem InfFlex, este apresenta propriedades características dos infinitivos de controlo?

Observa-se, em geral, que os aprendentes de PE L2 desenvolvem conhecimento de que o infinitivo flexionado, em contextos de controlo, apresenta propriedades características de infinitivos de controlo. Os resultados sugerem que, à semelhança dos falantes nativos, os aprendentes de PE atribuem preferencialmente leituras de controlo aos sujeitos nulos dos infinitivos, quer flexionados quer não flexionados, com os verbos lamentar, preferir e querer: controlo exaustivo, na condição de sujeito plural, e controlo parcial, na condição de sujeito singular. Como referimos acima, as taxas de seleção de leitura de referência livre nestes contextos são baixas.

A exceção ocorre com o verbo prometer: independentemente do número do sujeito matriz (singular ou plural), o sujeito da infinitiva tem preferencialmente uma leitura de antecedente split, tanto para falantes nativos como não-nativos. Fatores pragmáticos poderão estar na origem desta preferência, uma vez que os verbos utilizados na oração infinitiva são predicados coletivos, que denotam atividades que envolvem todos os indivíduos referidos. 


\section{Observa-se um efeito de L1 na aquisição destas propriedades?}

Ambos os grupos de L1 apresentam um percurso de desenvolvimento idêntico, existindo diferenças apenas com os verbos querer e preferir, isto é, os verbos com os quais o InfFlex é menos aceitável na gramática do PE. Com estes verbos, na condição de singular, os grupos de espanhol L1 têm taxas de aceitação de InfFlex inferiores aos grupos de inglês L1 correspondentes e taxas de aceitação de infinitivo não flexionado superiores. Esta diferença poderá indiciar um efeito de influência da L1, constituindo, neste caso, a existência de infinitivo pessoal em espanhol um fator facilitador do desenvolvimento.

\section{Observa-se um efeito de desenvolvimento na aquisição destas propriedades?}

Aparentemente, sim, mas este efeito só será visível com o verbo preferir no grupo de inglês L1, que se traduz na diminuição da aceitação de InfFlex com este verbo no grupo avançado, e com o verbo querer no grupo de espanhol L1, em que também se observa uma diminuição da aceitação de InfFlex, mas apenas na condição de singular. O grupo de espanhol L1 apresenta também um aumento na aceitação de leitura de referência livre para o sujeito do InfFlex com o verbo lamentar (e também com preferir), aproximando-se, assim, do desempenho do grupo de controlo.

Apesar de se observar algum desenvolvimento dos grupos intermédios para os avançados, é evidente que o conhecimento das propriedades do InfFlex nestes contextos de controlo ainda não está plenamente adquirido nos grupos avançados, ao contrário do que tem sido observado relativamente às propriedades gramaticais do InfFlex standard, i.e., não controlado (Madeira, 2018). Porém, os resultados do presente estudo estão em linha com o que se sabe quanto ao desenvolvimento tardio das propriedades do InfFlex com verbos de controlo na aquisição de PE L1 (Santos, Gonçalves \& Hyams, 2016). Os aprendentes revelam dificuldades sobretudo na identificação dos verbos de controlo com que o InfFlex é possível apenas marginalmente (preferir e querer) e na determinação das propriedades deste infinitivo em cada um dos contextos (nomeadamente, em que contextos o InfFlex é obrigatoriamente um infinitivo de controlo e em que contextos permite sujeitos com referência livre).

O conhecimento das propriedades de seleção dos verbos situa-se na interface entre léxico e sintaxe. Estas propriedades de interface, mesmo tratando-se de interfaces internas, parecem ser problemáticas, o que poderá explicar o atraso no desenvolvimento das propriedades do InfFlex investigadas no presente estudo. Assim, este atraso pode ser atribuído ao facto de a aquisição destas propriedades depender, não apenas de conhecimento sintático, mas também de conhecimento lexical e da integração destes dois tipos de conhecimento.

As dificuldades poderão ainda dever-se ao facto de, em contextos de controlo, o InfFlex ocorrer em alternância com o infinitivo não flexionado (embora apresente, como vimos, uma distribuição mais restrita), exibindo os dois infinitivos propriedades idênticas (i.e., propriedades de controlo obrigatório), o que pode tornar problemática a distinção entre ambos para os falantes não-nativos.

Outro fator que poderá explicar as dificuldades observadas prende-se com questões de input linguístico. As construções de InfFlex com verbos de controlo de sujeito apresentam baixa frequência no input e a variabilidade que se observa nas gramáticas nativas poderá contribuir para que as evidências a que os aprendentes têm acesso não sejam suficientemente claras. Além disso, o facto de o InfFlex em estruturas de controlo de sujeito não ser objeto de ensino formal, ao contrário do InfFlex standard, poderá contribuir também para as dificuldades observadas.

Finalmente, a inexistência de infinitivos flexionados na L1 dos aprendentes pode constituir um fator de dificuldade adicional para a aquisição destas construções em PE L2, embora, como sugerimos, a existência do 
infinitivo pessoal na gramática da L1 seja potencialmente facilitadora desta tarefa de aquisição para falantes nativos de espanhol.

Mais investigação é necessária para perceber a natureza das dificuldades identificadas neste estudo. No futuro, pretendemos (i) alargar a amostra incluindo níveis mais avançados/quase-nativos e outras L1; (ii) testar outros verbos de controlo, incluir predicados não coletivos, e investigar outras propriedades de controlo (e.g., a impossibilidade de realização do sujeito infinitivo e a obrigatoriedade de um antecedente local em posição de c-comando) e outras propriedades do InfFlex. Independentemente das questões relacionadas com a aquisição, consideramos igualmente essencial explorar melhor a comparação com o infinitivo não flexionado e as potenciais diferenças entre os diferentes verbos nas gramáticas nativas.

\section{Referências:}

Agostinho, Celina (2014) The acquisition of control in European Portuguese complement clauses. Dissertação de Mestrado, Universidade de Lisboa.

Fiéis, Alexandra e Ana Madeira (2017) The Portuguese inflected infinitive across varieties. In P. Barbosa, M. C. Paiva e C. Rodrigues (orgs.) Studies on variation in Portuguese. Issues in Hispanic and Lusophone Linguistics 14. Amsterdam/Philadephia: John Benjamins, pp. 280-299.

Gonçalves, Anabela, Ana Lúcia Santos e Inês Duarte (2014) (Pseudo-)inflected infinitives and control as Agree. In K. Lahousse e S. Marzo (orgs.) Romance languages and linguistic theory. Amsterdam: John Benjamins, pp. 161-180.

Hornstein, Norbert (1999) Movement and control. Linguistic Inquiry 30, pp. 69-96.

Landau, Idan (2000) Elements of control. Structure and meaning in infinitival constructions. Dordrecht: Kluwer.

Madeira, Ana (2018) Inflected infinitives in L2 Portuguese. In A.L. Santos e A. Gonçalves (orgs.) Complement clauses in Portuguese: Syntax and acquisition. Amsterdam/Philadephia: John Benjamins, pp. 321-360.

Madeira, Ana e Alexandra Fiéis (2019) Inflected infinitives in Portuguese. In L. Franco e P. Lorusso (orgs.) Linguistic variation: Structure and interpretation. Contributions in honor of M. Rita Manzini. Studies in Generative Grammar 132. Berlin/New York: De Gruyter Mouton, pp. 423-437.

Mensching, Guido (2000) Infinitive constructions with specified subjects: A syntactic analysis of the Romance languages. Oxford: Oxford University Press.

Modesto, Marcello (2010) What Brazilian Portuguese says about control: remarks on Boeckx and Hornstein. Syntax 13 (1), pp. 78-96.

Pires, Acrisio (2001) The syntax of gerunds and infinitives: Subjects, case and control. Tese de Doutoramento, Universidade de Maryland.

Pires, Acrisio, Jason Rothman e Ana Lúcia Santos (2011) L1 acquisition across Portuguese dialects: Modular and interdisciplinary interfaces as sources of explanation. Lingua 121, pp. 605-622.

Raposo, Eduardo (1989) Prepositional infinitival constructions in European Portuguese. In O. Jaeggli e K. Safir (orgs.) The null subject parameter. Studies in Natural Language and Linguistic Theory, vol 15. Dordrecht: Springer, pp. 277-305.

Raposo, Eduardo (1987) Case Theory and Infl-to-Comp: The inflected infinitive in European Portuguese. Linguistic Inquiry 18, pp. 85-109.

Santos, Ana Lúcia, Anabela Gonçalves e Nina Hyams (2016) Aspects of the acquisition of object control and ECM-Type verbs in European Portuguese. Language Acquisition 23 (3), pp. 199-233.

Santos, Ana Lúcia, Jason Rothman, Acrisio Pires e Inês Duarte (2013) Early or late acquisition of inflected infinitives in European Portuguese? Evidence from spontaneous production data. In M. Becker, J. 
Grinstead e J. Rothman (orgs.) Generative linguistics and acquisition: Studies in honor of Nina M. Hyams. Amsterdam/Philadephia: John Benjamins, pp. 65-88.

Sheehan, Michelle (2018) Control of inflected infinitives in European Portuguese. In A.L. Santos e A. Gonçalves (orgs.) Complement clauses in Portuguese: Syntax and acquisition. Amsterdam/Philadephia: John Benjamins, pp. 27-58.

Sheehan, Michelle (2014) Portuguese, Russian and the theory of control. In H. Huang, E. Poole e A. Rysling (orgs.) Proceedings of the 43rd annual meeting of the North East Linguistic Society 43 (NELS 43), vol. 2. Amherst, MA: GLSA, pp. 115-126.

Sorace, Antonella (2011) Pinning down the concept of 'interface' in bilingualism. Linguistic Approaches to Bilingualism 1, pp. 1-33.

Torrego, Esther (1998) Nominative subjects and pro-drop INFL. Syntax 1 (2), pp. 206-219. 